\title{
Role of the indoor environment in determining the severity of asthma
}

\author{
Ashley Woodcock, Adnan Custovic \\ North West Lung Centre, Wythenshawe Hospital, Southmoor Road, \\ Manchester M23 9LT, UK
}

Introductory articles

Allergen exposure decreases glucocorticoid receptor binding affinity and steroid responsiveness in atopic asthmatics

SR Nimmagadda, SJ Szefler, JD Spahn, W Surs, DYM Leung

Allergen exposure can confound the management of asthma. To understand the potential mechanisms by which allergens increase the steroid requirements in atopic asthmatics, we examined the effects of allergens on glucocorticoid receptor (GCR) binding affinity and glucocorticoid (GC) responsiveness of peripheral blood mononuclear cells (PBMC) from atopic asthmatics. A significant reduction $(p<0.001)$ in the GCR binding affinity $\left(\mathrm{K}_{\mathrm{d}}\right)$ was observed in ragweed-allergic asthmatics during ragweed pollen season compared with PBMC obtained before and after ragweed season. In vitro effects of allergen on PBMC GCR $\mathrm{K}_{\mathrm{d}}$ were also examined by incubating PBMC from atopic asthmatics with allergen (ragweed and cat) versus Candida albicans. GCR binding affinity was significantly reduced after incubation with ragweed $(p<0.001)$ or cat allergen $(p<0.001)$ compared with baseline or $C$. albicans stimulation. This effect was limited to atopic asthmatics in that in vitro cat allergen incubation for $48 \mathrm{~h}$ failed to significantly alter GCR binding affinity in nonasthmatic, atopic individuals. These allergen-induced redutions in GCR binding affinity also rendered the PBMC less sensitive to the inhibitory effects of hydrocortisone and dexamethasone on allergen-induced proliferation $(p<0.01)$. To test the hypothesis that allergen-induced alterations in GCR binding affinity were cytokine-induced, we examined the effects of interleukin-2 (IL-2) and IL-4 neutralization using anticytokine antibodies. Addition of both anti-IL-2 and anti-IL-4 antibodies resulted in a significant $(p<0.001)$ inhibition of allergen-induced alterations in GCR binding affinity. Furthermore incubation with cat allergen induced significantly higher concentrations of IL-2 $(p=0.03)$ and IL-4 $(p=0.02)$ by PBMC from atopic as compared with nonatopic subjects. Our current observations suggest that allergen exposure may contribute to poor asthma control by reducing GCR binding affinity in mononuclear cells. This appears to be mediated through IL-2 and IL-4. These findings may have important implications for novel approaches to the treatment of poorly controlled asthma. (Am J Respir Crit Care Med 1997;155:87-93)

Allergen reduction measures in houses of allergic asthmatic patients: effects of aircleaners and allergen-impermeable mattress covers

S van der Heide, HF Kauffman, AEJ Dubois, JGR de Monchy

Recommendations for allergen avoidance or allergen reduction measures play an important part in the treatment of allergic asthmatic patients. The purpose of this study was to test recently developed aircleaners with respect to their capacity to capture airborne allergen particles and to improve clinical parameters of asthmatic patients sensitized to aeroallergens. Forty five allergic asthmatic patients were studied in a double-blind procedure for 6 months. The patients were divided into three groups of 15 patients. In Group 1, the intervention consisted of the application of active air-cleaners in living-rooms and bedrooms. In Group 2, placebo air-cleaners were used in combination with allergen-impermeable mattress covers. In Group 3, the same intervention was performed as in Group 2 but with active aircleaners. Allergen levels in mattress and floor dust were measured before, and 3 and 6 months after the interventions. After 6 months, the air-cleaners were dismantled and the filters were analysed for the amount of dust collected and allergen content. Immunological and lung function parameters were 
measured before, and 3 and 6 months after the interventions. Considerable amounts of airborne dust and allergic particles were captured in the filters of the air-cleaners. Up to the $18.9 \mathrm{~g}$ of dust, 4,513 $\mathrm{ng}$ of house dust mite allergen, Der $p$ 1, and 50,000 $\mathrm{mU}$ of cat allergen, Fel $d 1$ (in houses with cats) were collected by air-cleaners in living-rooms. Only in Group 3 (in which both active air-cleaners and mattress covers were used) was a small (less than 1 doubling dose) but statistically significant improvement of provocative concentration of histamine causing a $20 \%$ fall in forced expiratory volume in one second $\left(P C_{20}\right.$ ) observed (from 5.96 to $9.02 \mathrm{mg} \cdot \mathrm{ml}^{-1}$ ). The amount of dust and house dust mite allergen collected in the filters was significantly correlated with an improvement of peak flow variation. In combination with other allergen avoidance measures, the examined air-cleaners can contribute to diminished allergen exposure and improvement of airway hyperresponsiveness in asthmatic patients. (Eur Respir J 1997; 10:1217-1223)

Asthma doctors tend to fall within a spectrum of two extremes, either enthusiasts for allergy or for pharmacotherapy. The former see asthma as an extrinsic problem which can be controlled by the strict avoidance of offending environmental insults or by immunotherapy. In their support, there are differences in the prevalence of asthma between the same genetic stock in differente evidence for increasing asthma prevalence with time. And the environment is changing; in addition to changes in ventilation, furnishings and house construction, in the UK there are now 15 million dogs and cats sharing our houses (more pets than children). On the other hand, the pharmacologically orientated physician sees asthma as an intrinsic airways disease involving whichever inflammatory cell and mediator is fashion inflammatory cell and mediator is fashionable this year 1980s PAF, and in the 1990s various cytokines and $T$ lymphocytes. Cynics compare asthma to an inflammatory goulash soup and argue that ever more detailed pea splitting will not help patients. Perhaps we should try to work out what is heating up the soup in the first placenamely, the environment. Whilst this is said tongue-incheek, there is more than a little truth in it.

However, we still need drugs to control established asthma. Glucocorticosteroids are the cornerstone of anti-inflammatory treatment of asthma. The therapeutic effect of steroids varies between patients with chronic asthma and is parallelled with a varying degree of glucocorticoreceptor (GCR) binding affinity in periphera blood mononuclear cells (PBMCs). ${ }^{1}$ Some asthmatic patients are relatively resistant to therapy with inhaled steroids. In many patients this may be due to poor compliance, but for other subjects this is a true drug compliance, but for other subjects this is a true drug
resistance which may in theory be inherited or acquired. resistance which may in theory be inherited or acquired. It has been known for some time that glucocorticoid
binding affinity is reduced in patients with steroid resistant asthma. ${ }^{2}$ This abnormality normalises after 48 hours in culture, but can be restored by co-incubation in a medium containing IL-4 and IL-2.

In addition, the airways of steroid resistant asthmatics are known to have higher IL- 2 and IL- 4 cytokine gene expression. ${ }^{2}$ This implies that the binding of steroids to PBMCs is influenced by cytokines. Identifying factors which are associated with steroid resistance would have important diagnostic and therapeutic implications.

Allergen exposure and steroid responsiveness The first introductory paper by Nimmagadda et al starts to bridge the gap between pharmacologists and allergists, and provides evidence that allergen exposure may confound the pharmacological management of the dis- ease. ${ }^{3}$ This group investigated the effect of allergen exposure on GCR binding affinity and responsiveness to glucocorticoids of PBMCs in atopic asthmatics both in vivo and in vitro. The effect of in vivo exposure was tested in patients allergic to ragweed before, during and after the ragweed pollen season. A significant reduction in GCR binding affinity was observed during the pollen season compared with pre-season and post-season measseason compared with pre-season and post-season measurements (fig 1). It is of interest that there was no difference between the patients on inhaled steroids and those on $\beta_{2}$ agonists only.

The effect of in vitro allergen exposure was also investigated by incubating PBMCs from atopic asthmatics with either the relevant allergen to which they were sensitised or Candida albicans (as a control). PBMCs of ragweed allergic asthmatics obtained outside the pollen season and of cat allergic patients not exposed to cats had significantly reduced GCR binding affinity after incubation with ragweed and cat allergen, respectively, compared with both baseline and Candida albicans stimulation. This confirms that the observed effect is allergen specific and is restricted to atopic asthmatic patients as no similar effect was found in atopic non-asthmatic individuals or non-atopic subjects. Furthermore, the allergen induced reduction in GCR Furthermore, the allergen induced reduction in GCR
binding affinity of PBMCs from atopic asthmatic subbinding affinity of PBMCs from atopic asthmatic sub-
jects made the lymphocytes significantly less responsive to the inhibitory effect of hydrocortisone.

Cat allergen may induce IL- 2 and IL- 4 production from PBMCs of sensitised individuals. The incubation

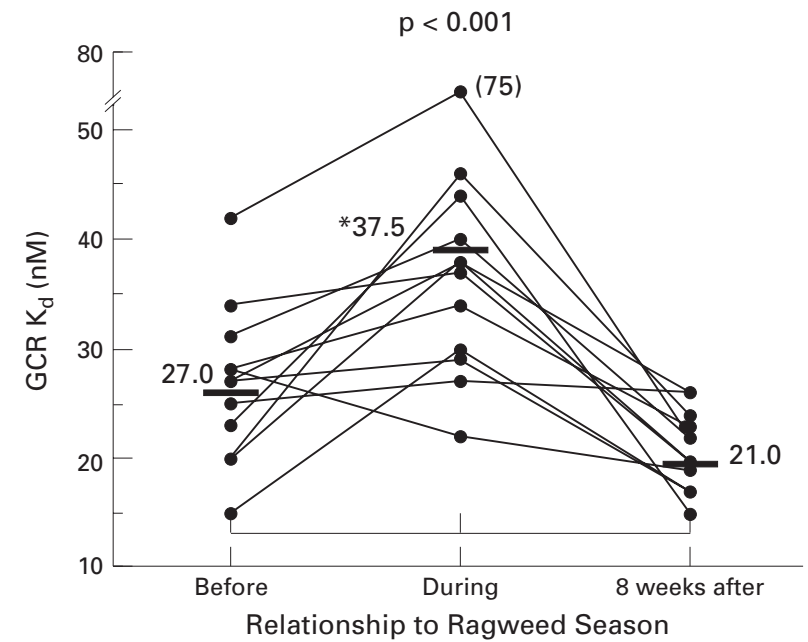

Figure 1 Effect of exposure to ragweed pollen on GCR binding affinity in patients allergic to ragweed. Reproduced 
of PBMCs from atopic asthmatics with cat allergen and either anti-IL2 or anti-IL4 antibodies inhibited the previously observed reduction in GCR binding affinity, suggesting that IL-2 and IL-4 may play an important role in altered steroid responsiveness, and that the effect of cat allergen ines. The finding that allergen exposure reduces GCR binding affinity in atopic asthmatics both in vivo and in vitro with the resulting functional alteration in cellular response to glucocorticoids supports the view that high allergen exposure in sensitised individuals may contribute to poor asthma control and maintenance of the inflammatory process in the airways, in spite of treatment with inhaled steroids.

Showing an effect of environment on inflammation or drug responsiveness is important, but what happens when patients try to reduce allergen exposure? In the past, many studies have looked at allergen exposure and allergen sensitisation separately as risk factors for the development and severity of asthma. Since house dust mites are the commonest source of allergens in temperate climates, we will look at the relationship between exposure to mites, atopic sensitisation, and asthma in more detail.

\section{Allergens and asthma}

The relationship between mites and asthma is complex and can be investigated with respect to: (1) exposure to mites and allergic sensitisation; (2) allergic sensitisation and asthma; (3) exposure to mites and asthma development; and (4) exposure to mites and asthma symptoms

EXPOSURE TO MITE ALLERGEN AS A RISK FACTOR FOR SENSITISATION

In a German Multicentre Atopy Study 1314 newborn babies were selected for a prospective study of the influence of exposure to indoor allergens on atopic sensitisation (499 at high risk; remaining 815 chosen at random). ${ }^{4}$ Both dust samples (for determination of allergen exposure) and blood samples (for determination of sensitisation) were available for 764 children. Children sensitised to dust mite had a significantly higher level of mite allergen in their homes than those in the non-sensitised group $(868 \mathrm{ng} / \mathrm{g}$ vs $210 \mathrm{ng} / \mathrm{g})$. In a recent non-sensitised group ( $868 \mathrm{ng} / \mathrm{g}$ vs $210 \mathrm{ng} / \mathrm{g}$ ). In a recent study in Linkoping (Sweden) Munir et al followed a group
of 86 children at high risk from birth until five years of of 86 children at high risk from birth until five years of age. $^{5}$ Eleven children developed sensitisation to indoor mites. The finding that only one of 86 children in this study was sensitised to mites during the first five years of life, despite a strong family history of allergic disease, may relate to the very low exposure to mite allergens (the highest level recorded was only $3.5 \mu \mathrm{g} / \mathrm{g}$ ). In a recent highest level recorded was only $3.5 \mu \mathrm{g} / \mathrm{g}$ ). In a recent
cross sectional study from Norway, sensitisation to mites cross sectional study from Norway, sensitisation to mites
correlated strongly with levels of mite allergen in mattresses correlated strongly with levels of mite allergen in mattresses
(corresponding to odds ratio (OR) of 16). ${ }^{6}$ Warner et a reported thang to odds ratio (OR) prevalence of sensitivity to dust mites and the level of mite specific IgE were both significantly associated with the concentration of mite allergen in homes.

Early infancy has been identified as a critical period for primary sensitisation. Evidence to support this view comes from studies relating atopy to month of birth Exposure to pets in early infancy is associated with specific IgE sensitisation and allergic disease later in childhood. Exposure to Der $p 1$ at a level of $>2 \mu \mathrm{g} / \mathrm{g}$ dust during infancy is associated with increased prevalence of positive skin tests and increased concentrations of $\operatorname{IgE}$ specific to dust mite by the age of five in children of atopic parents. ${ }^{8}$

SENSITISATION TO MITE ALLERGEN AS A RISK FACTOR FOR ASTHMA

On the east coast of the USA sensitisation to mite allergens is the single strongest risk factor for asthma among middle school children. ${ }^{9}$ Wickman et al reported that even in an area with low mite allergen exposure (Sweden) there was a close association between mite sensitisation and asthma (OR 4.9) ${ }^{10}$ Leung et al have to allergens was a significant risk factor for asthma in Chinese populations living in three different areas of south-east Asia, with adjusted ORs for mite sensitisation being between 1.9 and $5.8 .^{11}$ In a group of 68 children of atopic parents prospectively followed from birth in Poole, Dorset, sensitisation to mites at the age of 10 years was associated with an OR of 19.7 for having asthma. ${ }^{12}$

EXPOSURE TO MITE ALLERGEN AS A RISK FACTOR FOR ASTHMA

A recent study of children from six regions of New South Wales, Australia with a range of levels of mite allergens has provided further strong evidence on the role of exposure to mite allergens in childhood asthma ${ }^{13}$ ive to mites in each of the areas were at significant risk for having asthma, with the risk of mite sensitised children having asthma doubling for every doubling of the current level of exposure to mite allergens. In a previously mentioned study from Poole, exposure to Der p 1 at a level of $>10 \mu \mathrm{g} / \mathrm{g}$ measured in infancy was associated with a 4.8 fold relative risk of developing atopic asthma at the age of $11 .{ }^{12}$

EXPOSURE TO MITE ALLERGEN AND ASTHMA SYMPTOMS It is difficult to demonstrate the dose-response relationship between exposure to indoor allergen and asthma severity. Many asthmatic patients are sensitised and exposed to more than one allergen, and the relative to elucidate "Trigger factors" such as viruses, air pollution, exercise, and emotion, may make asthma severity worse. In addition, some sensitised patients react to a very low dose of allergen, whilst in others the level required to cause symptoms is considerably higher. Nevertheless, when Peat et al compared children living in areas with different levels of exposure to mites, they found a similar prevalence of mite sensitivity in both regions, but more severe bronchial hyperreactivity in sensitised children in the area with the highest mite levels. ${ }^{14}$ We have found a modest but significant correlation between the concentration of Der $\mathrm{p} 1$ in the bed and objective indices of asthma severity (non-specific bronchial hyperreactivity, $\mathrm{FEV}_{1}$ and PEF variability) in mite sensitised adult asthmatics, but not for patients with negative skin prick tests to mites ${ }^{15}$

We have recently shown that exhaled nitric oxide (NO), a marker of bronchial inflammation, is increased in asthmatics who are both sensitised and exposed to the relevant allergen compared with those who are sensitised but not exposed. ${ }^{16}$ In addition, patients with more severe asthma, and especially those with brittle asthma, are both sensitised and exposed to high levels of pet allergens. ${ }^{17}$ 
On the basis of current evidence it seems likely that sensitised patients exposed to high levels of allergen will usually have more severe disease than those exposed to low levels of allergens and that they should benefit from the effective environmental control.

\section{Allergen avoidance}

Effectiveness of allergen avoidance in the treatment of asthma was first suggested in studies in which patients were removed from their homes containing high levels of mite allergen into environments where allergen levels were low. In Europe the mite population density is very low at high altitudes $(>1500 \mathrm{~m})$ where humidity is insufficient to support mite survival. A number of studies insufficient to support mite survival. A number of studies conducted at high altitude sanatoria (e.g. Davos, Mis-
urina) have demonstrated an improvement in non-speurina) have demonstrated an improvement in non-spe-
cific bronchial hyperresponsiveness and symptoms with cific bronchial hyperresponsiveness and symptoms with re-exposure resulting in a rapid relapse. ${ }^{18}$ The high altitude studies were not controlled and it is possible that other domestic factors such as pets, environmenta tobacco smoke, or indoor pollution contributed to the observed improvement in asthma control. The lesson from high altitude studies is that it is essential to achieve and maintain a major reduction in allergen levels, and that even with such a reduction in exposure it may take many months for the improvement in asthma control to become fully apparent.

The important practical question is whether allergen levels in homes can be reduced sufficiently to improve asthma control in sensitised patients. We have recently asthma control in sensitised patients. We have recently
reviewed the practical measures for allergen avoidance. ${ }^{18}$ reviewed the practical measures for allergen avoidance. ${ }^{18}$ There are conflicting data on the effectiveness of allergen avoidance carried out in houses, primarily because the majority of studies have been small, poorly controlled, and have often used measures that do not reduce mite allergen exposure. Consequently, many fail to show clinical benefits and, as a result of low power, it is not clear which subgroups of patients will benefit most from the reduction in allergen exposure. Double blind, from the reduction in allergen exposure. Double blind,
placebo controlled trials of allergen avoidance are placebo controlled trials of allergen avoidance are
difficult to conduct. Almost every controlled study has observed a significant reduction in mite allergen levels and sometimes improved clinical symptoms in both the control group as well as the active group. ${ }^{1}$

The second introductory paper by van der Heide et $a l$ highlights the difficulties faced by investigators when conducting studies of allergen avoidance. ${ }^{19}$ In this trial a relatively small number (45) of house dust mite sensitive adults with mild asthma were randomised into one of the three groups: group 1, active air cleaners in living rooms and bedrooms; group 2, placebo air cleaners and mattress and pillow covers; group 3, active air cleaners and mattress and pillow covers. Unfortunately, in addition to house dust mite, most of the patients were also sensitised to other inhalant allergens ( 22 to pollens also sensitised to other inhalant allergens ( 22 to pollens
and cats; four of the cat allergic patients lived in a home with a cat). Mattress covers were once again shown to be very effective in reducing the amount of mite allergen Der $\mathrm{p} 1$. Unexpectedly, the placebo air cleaners (essential to prevent unblinding of patients and investigators) also captured dust and allergens on the coarse filter. Using changes in the airway hypercoarse filter. Using changes in the airway hyper-
responsiveness as a primary outcome measure, in the responsiveness as a primary outcome measure, in the
multiple regression analysis the authors have shown that multiple regression analysis the authors have shown that the greatest improvement in $\mathrm{PC}_{20}$ histamine was found in patients who had textile floor covering in the living largest decrease in the concentration of mite allergen Der $\mathrm{p} 1$ in mattress dust.
This study raises two important questions, unfortunately without providing the answer to them: (1) what are the most appropriate avoidance measures for individual patients with asthma and (2) who are the patients who will benefit most from allergen avoidance?

WHAT ARE THE MOST APPROPRIATE AVOIDANCE

MEASURES FOR INDIVIDUAL PATIENTS WITH ASTHMA? Mite allergens can be detected in the air in significant amounts only after vigorous disturbance ${ }^{20}$ and are predominantly contained within relatively large particles $(>10 \mu \mathrm{m}$ diameter). We deduce that most mite allergen exposure is from bedding, and that bed covering ought to be an effective control measure. In contrast, cat and dog allergens are readily measured in the air and $\sim 25 \%$ of airborne Fel $\mathrm{d} 1$ and Can $\mathrm{f} 1$ is associated with small particles $(<5 \mu \mathrm{m}$ diameter $) .{ }^{2122}$ This would imply that air filtration units have little place in mite avoidance but may be useful in removing cat and dog allergens from the air (this may be the reason why pet owners in van der Heide's study improved more than non-pet owners). Maybe the best approach in the future would be to think about integrated measures for avoiding all indoor allergens to which patients are sensitised rather than concentrating on a single allergen.

WHO ARE THE PATIENTS WHO WILL BENEFIT MOST FROM ALIERGEN AVOIDANCE?

It would appear that the patients benefiting most from allergen avoidance were those exposed to the highest levels of allergens before intervention (it is likely that these were the ones with the largest reduction in mite allergen levels; in addition, it is possible that the presence of carpets in living rooms could have been a marker of high allergen exposure as carpeted homes contain much carpets). This underlines the importance of individual measurements of exposure in sensitised asthmatic patients and stresses the need for a simple, cheap and effective way of assessing individual exposure. Novel semi-quantitative monoclonal antibody based immunodot strip techniques for the detection of house dust mite, cat, and cockroach allergen are currently assessing individual exposure in the near future.

Finally, is it mild asthmatics or those with severe disease who will benefit most from the effective avoidance of relevant allergens? The study by Nimmagadda et al suggests that patients with poorly controlled asthma and steroid resistant asthma are the ones who would benefit most from allergen reduction (but only to the airway inflammation with consequent improvement in nonspecific bronchial hyperresponsiveness and symptoms, and in improving their response to treatment with glucocorticosteroids). One of the reasons behind the spectacular results obtained in high altitude sanatoria could be that it is usually more severe patients who are referred to these centres rather than relatively milder asthmatics used in trials of allergen avoidance at home (e.g. the $\mathrm{PC}_{20}$ histamine in the previously reviewed study by van der Heide was $\sim 7 \mathrm{mg} / \mathrm{ml}$ ). ${ }^{19}$

Conclusions: allergen avoidance in tertiary

prevention of asthma

The cost of asthma care is increasing. It seems likely 
LEARNING POINTS

* Allergen exposure has been shown to reduce glucocorticoid receptor binding affinity and glucocorticoid responsiveness of peripheral blood mononuclear cells in atopic asthmatic subjects.

* This link may be important in explaining reduced efficacy of steroid anti-inflammatory therapy in some asthmatic subjects.

* This study is important as it demonstrates a potential link between environmental allergen exposure and pharmacotherapy in asthma.

* The relation between allergen exposure and asthma is complex and multi-faceted.

* Studies of house dust mite avoidance on incidence and severity of asthma are methodologically difficult and have produced conflicting data.

* Mite avoidance may become an important component in the management strategy of asthma following further well designed studies.

that an integrated approach which combines anti-inflammatory treatment (usually with an adequate dose of inhaled steroid) with environmental control of the relevant allergens (those to which the patient is sensitised) will be the most successful. Since mite sensitisation and exposure are most relevant in temperate climates, an effective, simple and widely applicable environmental intervention could have major benefits. While the overall effect of an intervention may be small at an individual level, in population terms this could still translate into clinically important and worthwhile health benefits. A randomised, blinded and controlled trial of house dust mite avoidance for one year is about to commence in the UK with 2000 enrolled asthmatic patients. Only studies such as this with adequate power will show which patients (and in which domestic environments) will benefit from the intervention. The results of such trials, along with long term studies of primary allergen avoidance, are awaited with interest.

1 Spahn JD, Leung DYM, Surs W, et al. Reduced glucocorticoid binding affinity in asthma is related to ongoing allergic inflammation. $A m$. Respir Crit Care Med 1995;151:1709-14.
Sher ER, Leung DYM, Surs W, et al. Steroid resistant asthma: cellular Sher ER, Leung DYM, Surs $\mathrm{W}$, et al. Steroid resistant asthma: cellular
mechanisms contributing to inadequate response to glucocorticoid therapy. 7 Clin Invest 1994; $93: 33-9$.

3 Nimmagadda SR, Szefler SJ, Spahn JD, et al. Allergen exposure decreases glucocorticoid receptor binding a affinity and steroid re155:87-93. Wahn U, Lau S, Bergmann R, et al. Indoor allergen exposure is a risk
factor for sensitisation during the first three years of life. $f$ Allergy Clin Munir AKM, Kjellman NIM, Bjorksten B. Exposure to indoor allergens in early infancy and sensitization. $\mathcal{F}$ Allergy Clin Immunol 1997;100 Dotterud LK, Van TD, Kvammen B, et al. Allergen content in dust
from homes and schools in northern Norway in relation to sensitisation and allergy symptoms in schoolchildren. Clin Exp Allergy 1997;27: 7 Warner AM, Bjorksten B, Munir AKM, et al. Childhood asthma and
exposure to indoor allergens: low mite levels are associated with exposure to indoor allergens: low mite levels are associated with 8 Rowntree S, Cogswell JJ, Platts-Mills TAE, et al. Development of IgE and IgG antibodies to food and inhalant allergens in children at ris of atopic disease. Arch Dis Child 1985;60:727-35.
Squillace SP, Sporik RB, Rakes G, et al. Sensitisation to dust mites as a dominant risk factor for asthma among adolescents living in Central
Virginia Virginia. Multiple regression analysis of a population-based study. $A m$
O Respir Crit Care Med 1997:156:1760-4 $\mathcal{F}$ Respir Crit Care Med 1997;156:1760-4.
Wickmann M Nordvall SL Pershat

10 mites in a cold temperate region. Am Rev Respir Dis 1993;148:58-62 Leung R, Ho P, Lam CWK, et al.. Sensitisation to inhaled allergens as

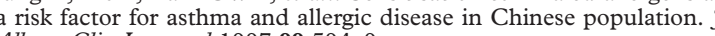

12 Sporik R, Holgate S, Platts-Mills TAE, et al.. Exposure to house dust mite allergen (Der pI) and the development of asthma in childhood. N Engl Med $1990,323.5027$

risk factor for childhood asthma in Australia. Am ₹ Respir Crit Care

Med 1996;152:144-6.
Peat JK, Tovey E, Gray EJ, et al. Asthma severity and morbidity in a pouse dust mite allergens. Aust NZ F Med 1994;24:270-6

Custovic A, Taggart SCO, Francis HC, et al. Exposure to house dust 16 Simpson A, Custovic A, Adisesh A, et al. Exhaled nitric oxide (eNO) in relation to sensitisation and exposure to indoor allergens in asthmatics. $f$ All $\mathrm{T} y \mathrm{Ch}$

17 exposure in brittle asthma. Am $\mathcal{H}$ Respir Crit Care Med 1996;153:A514 18 Custovic A, Simpson A, Chapman MD, et al. Allergen avoidance in

19 van der Heide S, Kauffiman HF, Dubois AEJ. et al. Allergen reduction measures in houses of allergic asthmatic patients: effects of air cleaners
and allergen-impermeable mattress covers. Eur Respir $\mathcal{F}$ 1997;10: 20 house dust mite allergens. I Allergy Clin Immunol 1995;96:134-5.

1 Custovic A, Green R, Fletcher A, et al. Aerodynamic properties of the major dog allergen, Can $\mathrm{f} 1$ : distribution in homes, concentration and
particle size of allergen in the air. Am $₹$ Respir Crit Care Med 1997;

$155: 94-8$. Smith A, Pahdi $\mathrm{H}$, et al. Distribution, aerodynamic char-
22 Custovic A, Smater
acteristics and removal of the major cat allergen Fel d 1 in British acteristics and removal of the major cat allergen Fel d 1 in British 